Military Technical College

Kobry El-Kobba

Cairo, Egypt

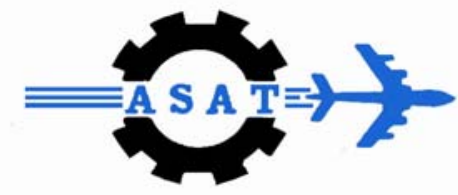

12-th International Conference

on

Aerospace Sciences \&

Aviation Technology

\title{
SPACECRAFT ATTITUDE AND ATTITUDE RATE ESTIMATION USING HYBRID KALMAN FILTERING OF MAGNETOMETER MEASUREMENTS
}

TAMER* M. H., SAYED** D.H., and GAMAL ${ }^{* * *}$ E.

\begin{abstract}
The primary goal for this work is to establish a three-axis spacecraft attitude estimator suitable for coarse attitude estimation and control during low accuracy spacecraft operational modes. An innovative algorithm for spacecraft attitude and angular velocity estimation is developed based on a new Kalman filtering approach applied to magnetometer measurements only and named as hybrid Kalman filtering. The new approach alleviates many problems encountered with the standard extended Kalman filter. It also estimates spacecraft attitude and attitude rate taking into account various sources of errors related to measurement and process noise. The filter could start with initial attitude and rate estimation errors as large as $180^{\circ}$ and $0.03 \%$ s respectively and still be able to converge to a $(3-\sigma)$ attitude and rate estimation errors of $0.8^{\circ}$ and 0.0017 $\%$ respectively. The new approach could be expanded to deal with other vector measurements besides the earth's magnetic field as well.
\end{abstract}

\section{KEYWORDS:}

Attitude, Attitude rate, estimation, quaternion, hybrid Kalman filter, magnetometer, disturbances, noise.

\footnotetext{
*PhD student, Aerospace Department, Cairo University, Guiza, Egypt

** Professor, Aerospace Department, Cairo University, Guiza, Egypt

${ }^{* * *}$ Professor, Aerospace Department, Cairo University, Guiza, Egypt.
} 


\section{INTRODUCTION}

For remote sensing purposes low-cost missions have attracted a great deal of interest. One way to reduce mission cost is to use low-cost sensors. Also, during coarse attitude spacecraft operational modes a need arise to estimate roughly the spacecraft attitude based on a minimum number of continuously, and low-power operating sensors. So magnetometers represent usually low-cost, high reliability, continuous operation, and low-power solutions suitable for such requirements.

The problem of spacecraft attitude determination is addressed in several ways in the literature. Traditional attitude determination algorithms such as the triad [1], the modified triad [2], and the q-method utilize measurements of at least two non-parallel vectors. While as Estimation algorithms could use even single vector measurements to estimate spacecraft attitude because the usually benefit from modeling the spacecraft attitude dynamics to reduce the minimum number of the required vectors.

Researches about the problem of spacecraft attitude and attitude rate estimation based on magnetometer data only have been found in the literature. The algorithms developed in this paper could deal with a range of spacecraft angular velocities comparable to those found in [3] without incorporating any measurements other than the readings of the magnetometer. The maximum initial attitude estimation error presented in [4] and [5] was about $60^{\circ}$ and $50^{\circ}$ respectively. Our proposed algorithm could deal with initial attitude estimation error as large as $180^{\circ}$ and achieve a steady state estimation error value of $0.27^{\circ}(1-\sigma)$.

In the following sections, a novel estimation algorithm is also presented. The proposed algorithm, which is named as hybrid Kalman filter (HKF), alleviates the brute-force linearization problems usually encountered with the standard extended Kalman filter and requires a new formulation of spacecraft dynamics and measurement equations. Despite the complexity of the problem at hand, stability of the new filter during long periods of operation and over a wide range of sampling frequencies is considered to be a vital advantage. Although being developed mainly to work with magnetometer measurements, the newly developed methodology could be expanded to deal with many other vector measurements such as the sun vector, the earth vector, the moon vector, and even stellar vectors as well.

An important subject that is to be addressed in this research is the generality of the extended Kalman filter which must be narrowed to deal with problem at hand. The traditional Kalman and extended Kalman filters don't guarantee that the estimated states remain within their permitted or logical values. For example, correcting a priori estimate of the state with the measurements to obtain posteriori state estimate doesn't guarantee that the posteriori state estimate satisfies the mathematical bounds of the states. One of such mathematical bounds is the unity magnitude of the quaternion vector which mustn't be violated by the measurement update process if the quaternion vector components 
are selected as states. In another words, the formulation of the Kalman and extended Kalman filters is a general one that doesn't assure the unity magnitude of the estimated quaternion.

\section{MODELING THE SPACECRAFT ROTATIONAL MOTION}

The kinematics of spacecraft attitude is represented by the well known relation

$$
\dot{Q}=\frac{1}{2} \Omega_{B R} Q
$$

Where $Q$ is the attitude quaternion vector $\left(Q=\left[\begin{array}{llll}q_{1} & q_{2} & q_{3} & q_{4}\end{array}\right]^{T}\right)$ describing the rotation from the orbit reference frame [6] to the body frame, $\Omega_{B R}$ is the $4 \times 4$ skewsymmetric matrix whose elements are the components of the spacecraft body axes angular velocity vector with respect to the orbit reference axes. Differentiation of Eq. (1) with respect to time gives

$$
\ddot{Q}=\frac{1}{2} \dot{\Omega}_{B R} Q+\frac{1}{2} \Omega_{B R} \dot{Q}
$$

We should note that $\dot{\Omega}_{B R}$ is the $4 \times 4$ skew-symmetric matrix form of $\dot{\omega}_{B R}$ which is the angular acceleration of the spacecraft body axes with respect to the orbit reference axes. The relation between the angular velocity vectors $\omega_{B R}$, and $\omega$ is governed by

$$
\omega_{B R}=\omega+D_{R}^{B}(Q)\left[\begin{array}{c}
0 \\
\omega_{0} \\
0
\end{array}\right]
$$

With $\omega$ defined as the inertial angular velocity vector of the spacecraft body axes with respect to the inertial frame of reference expressed in the body axes, $D_{R}^{B}(Q)$ is the spacecraft attitude matrix that transforms a vector from the orbit reference frame to the body frame, and $\omega_{0}$ is the spacecraft orbital angular velocity. Differentiation of Eq. (3) w.r.t. time gives

$$
\dot{\omega}_{B R}=\dot{\omega}+\dot{D}_{R}^{B}\left[\begin{array}{c}
0 \\
\omega_{0} \\
0
\end{array}\right]+D_{R}^{B}\left[\begin{array}{c}
0 \\
\dot{\omega}_{0} \\
0
\end{array}\right]
$$

Taking into account that 


$$
\dot{D}_{R}^{B}=-\left[\omega_{B R} \times\right] D_{R}^{B}
$$

And

$$
\dot{\omega}=I^{-1}[-\omega \times I \omega]+I^{-1} T
$$

Where $\left[\omega_{B R} \times\right]$ is the skew-symmetric cross-product equivalent matrix, $T$ is the torque acting on the spacecraft, $I$ is the spacecraft inertia matrix. Now, direct substitution from equations (5), and (6) into Eq. (4) gives

$$
\dot{\omega}_{B R}=I^{-1}[T-\omega \times I \omega]-\frac{1}{2}\left[\omega_{B R} \times\right] D_{R}^{B}\left[\begin{array}{c}
0 \\
\omega_{0} \\
0
\end{array}\right]+D_{R}^{B}\left[\begin{array}{c}
0 \\
\dot{\omega}_{0} \\
0
\end{array}\right]
$$

Thus, $\quad \dot{\omega}_{B R}$ could be now used to compute directly $\dot{\Omega}_{B R}$ in Eq. (2). Eq. (2) now is already setup for the newly developed HKF algorithm. Analyzing Eq. (2) carefully we could easily see that it has a set of advantages. First, it has included the body acting torque; $T$. Existence of this term in the equation enabled the modeling process of any disturbing torque without changing the structure of the filter this is because all what we care about are the values of the torque, $T$. The second advantage of Eq. (2) is the existence of a great deal of matching between the filter and the true dynamics due to the absence of any forcing function.

\section{3- MODELING THE MEASUREMENT PROCESS}

In this paper, the magnetometer is assumed to be the only available attitude sensor. The components of the earth's magnetic field vector in the orbital reference coordinate system, $b_{R}$, is related ideally to the vector of magnetometer measurements, $b_{B}$, by the relation

$$
b_{B}(Q)=D_{R}^{B} b_{R}
$$

Eq. (8) could be derived for the quaternion vector describing the rotation from the estimated plant to the real one [7]. This methodology has necessitated the change of some system states. In our case, system states had been selected (as shown in subsequent sections) and so it is required to develop a suitable measurement equation. 
The derivation starts by linearizing Eq. (8) about the estimated quaternion, $\hat{Q}$. Consequently, application of first order Taylor series expansion results in

$$
b_{B}(Q)=b_{B}(\hat{Q}+\Delta Q)=b_{B}(\hat{Q})+\left.\frac{\partial b_{B}(Q)}{\partial Q}\right|_{\hat{Q}}(Q-\hat{Q})
$$

Rearranging

$$
b_{B}(Q)-b_{B}(\hat{Q})+\left.\frac{\partial b_{B}(Q)}{\partial Q}\right|_{\hat{Q}} \hat{Q}=\left.\frac{\partial b_{B}(Q)}{\partial Q}\right|_{\hat{Q}} Q
$$

With the definition of $0_{4 \times 4}$ as a $4 \times 4$ zero matrix, the measurement equation becomes

$$
z=b_{B}(Q)-b_{B}(\hat{Q})+\left.\frac{\partial b_{B}(Q)}{\partial Q}\right|_{\hat{Q}} \hat{Q}=\left.\frac{\partial b_{B}(Q)}{\partial Q}\right|_{\hat{Q}} Q
$$

which is valid for any values of the estimated attitude quaternion vector. The partial derivative $\partial b_{B} / \partial Q$ is a matrix with linear algebraic elements. These elements are continuous (non-singular) algebraic functions of the quaternion vector components. This implies the existence of the matrix elements for any values of the quaternion vector components even if the estimated quaternion is very far away from the real one. We should also note that Eq. (11) isn't restricted to small Euler angles. The latter two points have extended the ability of the HKF to deal with large initial attitude estimation errors. Inclusion of the constant bias error, $b$, transforms Eq. (11) to

$$
z_{m}=b_{B}(Q)-b_{B}(\hat{Q})+\left.\frac{\partial b_{B}(Q)}{\partial Q}\right|_{\hat{Q}} \hat{Q}+b=\left.\frac{\partial b_{B}(Q)}{\partial Q}\right|_{\hat{Q}} Q+b
$$

The measurement process of the magnetic field vector is subject to a wide variety of errors. The first kind of errors is associated with the modeling process of the earth's magnetic field. The earth's magnetic field is subject to secular, temporal, diurnal variations. These variations aren't predicted by the international geomagnetic reference field (IGRF) model. In addition, magnetic storms could affect greatly the measured values of the earth's magnetic field occasionally. As a consequence, the earth's 
magnetic field could be modeled by the IGRF model with a prescribed accuracy of about $50 \mathrm{nT}$ [8]. The second kind of errors is related directly to the measurement process of the earth's magnetic field in the spacecraft body axes. The measured magnetic field is a function of a set of variables. These variables are:

1- Magnetometer bias.

2- Linear scale factor error matrix $\left(S_{c}\right)$.

3- Axes mounting error matrix $\left(N_{m}\right)$.

So as to include all these effects, the actual readings of the magnetometer, $b_{a}$, are represented by the relation

$$
b_{a}=b_{B}+b+S_{c} b_{B}+N_{m} b_{B}
$$

\section{4- HKF ALGORITHM}

\section{A. State Vector Selection}

The system states are selected to be

$$
X^{T}=\left[\begin{array}{lll}
Q & \dot{Q} & b
\end{array}\right]^{T}
$$

Although the selected states aren't conventionally used in the literature, they have the following benefits:

1- The maximum absolute value of a quaternion vector component is equal to 1 . This consecutively limits the maximum possible attitude quaternion error to \pm 1 .

2- The quaternion vector magnitude is always equal to 1 . Consequently, a normalization process could be done if this condition is violated due to computational errors.

3- Limiting the quaternion vector components to \pm 1 and the quaternion vector magnitude to 1 sets an upper limit value of the quaternion vector time derivative, $\dot{Q}$, and its magnitude, $\|\dot{Q}\|$.

4- Limiting the values of $\dot{Q}$, and $\|\dot{Q}\|$ restricts in turn the values of $\ddot{Q}$, and $\|\ddot{Q}\|$.

As a result of these benefits, the HKF input and output states are bounded to an upper bound. 


\section{B. State-Space Formulation}

The state space form of the nonlinear spacecraft model could now be written as

$$
\stackrel{o}{X}=\left[\begin{array}{c}
\dot{Q} \\
\ddot{Q} \\
\dot{b}
\end{array}\right]=\left[\begin{array}{ccc}
0.5 \Omega_{B R} & 0_{4 \times 4} & 0_{4 \times 3} \\
0.5 \dot{\Omega}_{B R} & 0.5 \Omega_{B R} & 0_{4 \times 3} \\
0_{3 \times 4} & 0_{3 \times 4} & 0_{3 \times 3}
\end{array}\right]\left[\begin{array}{c}
Q \\
\dot{Q} \\
b
\end{array}\right]=[F(X)] X=f(X)
$$

And the measurement matrix is

$$
Z_{m}=\left[\left.\frac{\partial b_{b}^{b}(Q)}{\partial Q}\right|_{\hat{Q}} 0_{3 \times 4} \quad I_{3 \times 3}\right] X=H X
$$

Equations (15), and (16) are considered to be the ideal relations of the plant and the measurement processes. In order to simulate the real world, uncertainties in the real plant (such as inertia matrix uncertainties) and measurement processes must be added.

\section{HKF Structure}

The HKF idea stems directly from the Kalman filter, pseudo-linear Kalman filter and the extended Kalman filter concepts. It borrows the idea of linear measurements with respect to the actual states (not the perturbed states) from the Kalman filter. The second borrowed idea is coming from the pseudo-linear Kalman filter by disregarding the nonlinear state dynamics and treating it as a time varying system [9]. The final borrowed concept is the measurement process linearization encountered by the extended Kalman filter. The basic five equation of the Kalman filter are

$$
\begin{gathered}
\dot{\hat{X}}=\left.f(\hat{X})\right|_{\hat{X}=\hat{X}_{k-1}^{+}} \\
P_{k}^{-}=A_{k-1}\left(\hat{X}_{k-1}^{+}\right) P_{k-1}^{+} A_{k-1}^{T}\left(\hat{X}_{k-1}^{+}\right)+Q_{k-1} \\
K_{k}=P_{k}^{-} H_{k}^{T}\left(\hat{X}_{k}^{-}\right)\left[H_{k}\left(\hat{X}_{k}^{-}\right) P_{k}^{-} H_{k}^{T}\left(\hat{X}_{k}^{-}\right)+R_{k}\right]^{-1}
\end{gathered}
$$




$$
\begin{gathered}
\hat{X}_{k}^{+}=\hat{X}_{k}^{-}+K_{k}\left[z_{k}-\hat{z}_{k}\right] \\
P_{k}^{+}=\left[I-K_{k} H_{k}\left(\hat{X}_{k}^{-}\right)\right] P_{k}^{-}\left[I-K_{k} H_{k}\left(\hat{X}_{k}^{-}\right)\right]^{T}+K_{k} R_{k} K_{k}^{T}
\end{gathered}
$$

where $\hat{X}_{k}^{-}$is a priori state estimate at a time step $k, A_{k}$ is the state transition matrix, $\hat{X}_{k}^{+}$is a posteriori state estimate at a time step $k, P_{k}^{-}$is the time propagated estimation error covariance matrix at a time step $k, Q_{k}$ is the process noise covariance, $R_{k}$ is the measurement noise covariance, $z_{k}$, is the measurement vector, $\hat{z}_{k}$ is the estimated value of the measurements based on the estimated states (i.e., $\hat{z}_{k}=\left.z\right|_{Q=\hat{Q}}$ ), and $P_{k}^{+}$is a posteriori estimation error covariance at a time step $k$. The state transition matrix according to [10] is calculated from

$$
A_{k}\left(\hat{X}_{k}^{+}\right)=\left\{I+\left(F_{k}\left(\hat{X}_{k}^{+}\right)\right) \Delta T\right\}
$$

With $\Delta T$ defined as the sampling time interval and

$$
F_{k-1}\left(\hat{X}_{k-1}^{+}\right)=\left.f\left(\Omega_{B R}, \dot{\Omega}_{B R}\right)\right|_{X=\hat{X}_{k-1}^{+}}
$$

The difference between the traditional extended Kalman filter and the HKF could be clearly explained with the help of equations (22) and (23). In the traditional extended Kalman filter, Eq. (22) is usually linearized with respect to time and states. Thus, $F_{k-1}\left(\hat{X}_{k-1}^{+}\right)=\partial f\left(\Omega_{B R}, \dot{\Omega}_{B R}\right) /\left.\partial X\right|_{X=\hat{x}_{k-1}^{+}}$. While as in the HKF (Which resembles the pseudolinear extended Kalman filter in its state transition matrix) Eq. (22) is linearized with respect to time only. Consequently, $F_{k-1}\left(\hat{X}_{k-1}^{+}\right)$is computed from Eq. (23).

\section{Singularity Inhibitors}

In order to use the spacecraft rotational motion derived model, a set of conditions should be satisfied. Some of these conditions are usually associated with the use of any quaternion vector to describe the spacecraft rotational motion and they are namely:

1- $\|Q\|=1$. This condition is usually expressed as a quaternion normalization process. 
2- $\|\dot{Q}\| \leq 2 / \Delta T$. This condition stems from the fact that the maximum value of any quaternion component is 1 . So the maximum difference between two successive quaternion vectors is expected to be 2. Accordingly, the maximum value of $\|\dot{Q}\|$ is restricted to $2 / \Delta T$.

3- $\|\ddot{Q}\| \leq(2 / \Delta T)^{2}$. This condition is a consequence of limiting the values of $\|\dot{Q}\|$.

These conditions could also be used to determine a guess of the maximum expected initial filter covariance matrix. The remaining set of conditions is coming from the Kalman filter formulation. This set is consisting from the following conditions

1- $\operatorname{det}\left(P_{k}^{-}\right)>0$. Where, det, stands for the matrix determinate operation.

2- $\operatorname{det}\left(P_{k}^{+}\right)>0$.

\section{E. Tuning Filter Parameters}

The development and behavior of any extended Kalman filter depends mainly on the knowledge of the covariance matrices, $P_{k}, Q_{k}$, and $R_{k}$ with a great deal of fidelity. Poor knowledge of these matrices could result in suboptimal performance, poor performance, divergence, or even singularity in the filter output. The off-diagonal terms in the covariance matrices represent the cross-correlation between the corresponding variables. For system with large numbers of states, the diagonal elements of the covariance matrices are usually selected by the designer based on his knowledge of the process and measurement noise [11]. Concatenation of this result with the first set of singularity inhibitors makes it reasonable to select $P_{0}^{+}$according to the relation

$$
P_{0}^{+}=\alpha P_{0}^{-}
$$

with $\alpha$, is a constant tuning parameter to be determined, and $P_{0}^{-}$is selected based on the first three conditions in the singularity inhabitance process. For example, to determine the value of $P_{0}^{-}$elements corresponding to the quaternion vector estimation error we could think of the estimated quaternion as a random number whose $(3-\sigma)$ maximum value is equal to 1 . So it is reasonable to select the standard deviation of any estimated quaternion error to be 0.3. This choice in turn limits the maximum values of the diagonal elements of the filter covariance matrix, ${P_{k}}^{+}$. And so on with the remaining components of $P_{0}^{-}$. Another important factor, that isn't usually treated in the literature, is 
the selection of the constant value, $\alpha$. In our case, this process is of a crucial importance. To obtain this value we've defined a loss function to be minimized, $J_{L}$, according to

$$
J_{L}=W\left\|b_{B}-\hat{b}_{B}\right\|+\gamma\|X-\hat{X}\|
$$

where $W$, and $\gamma$ are weighting coefficients. We should select the initial guess, $\alpha$, that minimizes the loss function, $J_{L}$. If the HKF is designed to select online the value of $\alpha$, then there is no means to measure the value of $\|X-\hat{X}\|$. Consequently, it is logical to let $\gamma=0$. On the other hand, consistency with Kalman filter problem formulation forces us to let $\gamma=1$, and $W=0$. This choice in turn means that the tuning process must be done off-line (either pre-flight or post-flight) and the true state vector value, $X$, is computed from simulation. Eq. (25) could also be used to select the best sampling time in addition to the constant, $\alpha$ by using a simple numerical two dimensional search algorithm. We should also notice that any value of $\gamma$ other than zero results in suboptimal or degraded performance of the filter due to the nonlinearity of the estimation and the measurement process. This means that there exists a set of solutions (of $\alpha, Q$, and $\dot{Q}$ values) that could minimize the loss function without being related to the true set of solution.

\section{F. Disturbance Modeling}

Ref. 1 represents the main source of computing various disturbances acting on the spacecraft which are namely: gravity gradient moment, magnetic disturbance moment, aerodynamic disturbance moment, and solar radiation moment.

In order to complete the derivation, a sun orbit simulator must be developed. In this simulator the sun is considered as if it were a satellite orbiting the earth with classical orbital elements : e (eccentricity) $=0.01675 \approx 0$, a (semi major axis) $=1.4949108 \mathrm{Km}$., $\mathrm{i}($ inclination $)=23.439^{\circ}, \theta_{\mathrm{S}}$ (true anomaly) $=0^{\circ}$ in the first day of spring, and $\Omega$ (right ascension of ascending node) $=0^{\circ}$. Additionally, the gravitational constant used in the simulation is considered to be the sun gravitational constant.

\section{G. Simulation Parameters, Results, and Testing}

A simulated spacecraft of low earth orbit is used to provide true reference data. The spacecraft orbit is assumed to be circular with an altitude of $800 \mathrm{Km}$ and an inclination of $98.6^{\circ}$. The earth's magnetic field model used was up to twelfth degree. Model coefficients are valid in the time span 2005-2010 as given in [12]. Model errors are assumed to have a standard deviation of 50nT. Magnetometer white noise is assumed to have a standard deviation of $3 \mathrm{mG}(300 \mathrm{nT})$. Magnetometer bias error has a value of 
$3 \mathrm{mG}$. The gravity gradient stabilized spacecraft has moments of inertia $I_{x}=80, I_{y}=82$ and $I_{z}=4 \mathrm{~kg}-\mathrm{m}^{2}$ with zero products of inertia.

The HKF proposed in our research has proved a superior performance. It could operate successfully over a wide range of sampling time intervals (actually it'd converge for sampling intervals ranging from 1 to 70 seconds) and for very long periods of operation as long as 25 orbits. Fig. 1 displays the average estimation error time history of the pitch, roll, and yaw angles over 20 realizations with different noise seeds. The time history shows that the filter is able to converge to the true solution despite the initial large estimation error which reaches a maximum value of $180^{\circ}$ initially. The estimation steady state error was about $0.6^{\circ}(3-\sigma)$ for the yaw angle, $0.8^{\circ}(3-\sigma)$ for the roll angle, and $0.7^{\circ}(3-\sigma)$ for the pitch angle. Although being initially as high as $0.03 \%$, the initial angular velocity estimation error decreases to a steady state error of about 0.0017 $\%(3-\sigma)$ as clarified by Fig. 2. Finally, as plotted in Fig. 3, the average total disturbing moment estimation error decreases as the filter converges to the true spacecraft attitude.

\section{H. Observability Analysis}

Observability analysis of the proposed system is borrowed directly from the linear control theory concepts. An advantage of this analysis is that system observability could be easily checked at each time step without substantial increase of the observability matrix size as given in [7]. Based on the earlier analysis we could put the system dynamics (excluding the bias states and dynamics) in several state space forms.

We could separate the equation of $\dot{Q}$ from the system defined by Eq. (15) and use the idea of reduced order estimator. Thus, the reduced order system is now defined by Eq. (1) with the measurement matrix

$$
Z_{R}(k)=\left.\frac{\partial b_{B}(Q)}{\partial Q}\right|_{\hat{Q}(k)} Q(k)
$$

The linear observability matrix could be defined at each time step as

$$
O_{1}(k)=\left[\begin{array}{c}
\partial b_{B}(Q) /\left.\partial Q\right|_{\hat{Q}(k)} \\
\partial b_{B}(Q) /\left.\partial Q\right|_{\hat{Q}(k)}\left[\Omega_{B R}(k-1) / 2\right] \\
\partial b_{B}(Q) /\left.\partial Q\right|_{\hat{Q}(k)}\left[\Omega_{B R}(k-1) / 2\right]^{2} \\
\partial b_{B}(Q) /\left.\partial Q\right|_{\hat{Q}(k)}\left[\Omega_{B R}(k-1) / 2\right]^{3}
\end{array}\right]
$$


Testing the rank of the observability matrix, $O_{1}$, has revealed that it has an initial rank of 3 that increases up to 4 (i.e., full rank) after two time steps as shown clearly in the first illustration of Fig. 4. The initial observability rank (which is 3 ) means that we are able to estimate only three components of the quaternion vector while indicating a trivial problem in the estimation of the remaining component. This trivial problem is totally alleviated because the remaining quaternion component could be computed from the quaternion normalization condition. If we used the system of equations defined by equations (1) and (2) along with the measurement matrix given by

$$
Z_{n}=\left[\left.\frac{\partial b_{b}^{b}(Q)}{\partial Q}\right|_{\hat{Q}} 0_{3 \times 4}\right] X
$$

The observability matrix, $\mathrm{O}_{2}$, rank is found to be the same as the latter rank. Taking into account that the reduced order estimator has proved the quaternion vector observability, we could easily deduce that the observable states of the system given by equations (1), and (2) are the quaternion vector components. Inclusion of the bias states and the bias dynamics into the system results in a full order estimation process with an initial observability matrix, $\mathrm{O}_{3}$, rank of 3 that increases up to 7 after two time steps as shown clearly in the third illustration of Fig. 4. Consequently, a considerable increase of the system observability that is reflected into smaller estimation error standard deviation is achieved.

\section{5- CONCLUSION}

The HKF has proved to be computationally effective for coarse attitude determination tasks. Complicated models of various disturbing torques could be used with the same filter structure. Filter stability over a wide range of sampling time intervals is a surplus advantage that could be used to minimize the total number of calculations required to estimate the spacecraft attitude along its orbit by increasing the sampling time.

Although filter measurement equation is derived mainly for earth's magnetic field measurements, it could be expanded easily to deal with many vector measurements such as sun or moon vector measurements. Using the quaternion vector and its time derivatives in the filter simplifies the selection process of optimal filter parameters in addition to inhibiting the filter from going into singularities. Large values of measurement errors didn't hold down the filter convergence. Steady state attitude and angular rate estimation errors of $0.8^{\circ}$, and $0.0017 \%(3-\sigma)$ respectively are also achievable. Finally, filter application to magnetometer measurements represents a feasible, low-cost, high reliability solution to the problem of coarse attitude determination. 


\section{6- ACKNNOWLEDGMENTS}

The first author thanks Dr., Mohammad I., Abdel-Rahman, and Dr., Ahmad Y., El-Rafay for their valuable discussions. The first author also thanks Prof. Mark L., Psiaki for his precious answers and assists.

\section{7- REFERENCES}

[1] Wertz, J. R., "Spacecraft Attitude Determination and Control", D. Reidel Publishing Company, 1997, Chaps. 12, 17.

[2] Habib, T., "The Global Positioning System Application to Satellite Position and Attitude Determination", M. Sc., Dissertation, Aerospace Department, Faculty of Engineering, Cairo University, Egypt, 2003.

[3] Azor, R., Itzhack Y. Bar-Itzhack, and Harman, R., "Satellite Angular Rate Estimation from Vector Measurements", Journal of Guidance Control and Dynamics, Vol.21, No.3, 1998, pp. 450-457

[4] Psiaki, M., Martel, F., and Pal, P., "Three Axis Attitude Determination via Kalman Filtering of Magnetometer Data", Journal of Guidance Control and Dynamics, Vol.13, No.3, 1990, pp. 506-514.

[5] Appel, P., "Attitude Estimation from Magnetometer and Earth-Albedo-Corrected Coarse Sun Sensor Measurements", Acta Astronautica, Vol., 56, 2005, pp. 115-126.

[6] Sidi, M. J., "Spacecraft Dynamics and Control, a Practical Engineering Approach", Cambridge University Press, 1997.

[7] Deutshmann, J., Itzhack Y. Bar-Itzhack, "Evaluation of Attitude and Orbit estimation Using Actual Earth Magnetic Field Data", Journal of Guidance Control and Dynamics, Vol.24, No.3, 2001, pp. 616-623.

[8] Makovec, K., "A Nonlinear Magnetic Controller for Three-Axis Stability of Nanosatellites", MSc thesis, Virginia Polytechnic Institute and State University, 2001.

[9] Abdel-Rahman, M. I., "Development and Application of New Attitude Control Algorithms for Spacecraft", Ph.D., Dissertation, Aerospace Department, Faculty of Engineering, Cairo University, Egypt, 2002.

[10] Shorshi, G., Itzhack Y. Bar-Itzhack, "Satellite Autonomous Navigation Based on Magnetic Field Measurements", Journal of Guidance Control and Dynamics, Vol.18, No.4, 1995, pp. 843-850.

[11] Powell, T., Martel, F., and Pal, P., "Automated tuning of an extended Kalman Filter Using the Downhill simplex Algorithm", Journal of Guidance Control and Dynamics, Vol.25, No.5, 2002, pp. 901-908.

[12] McLean, S., et al, "The US/UK World Magnetic Model for 2005-2010", NOAA Technical Report, NESDIS/NGDC-1. 

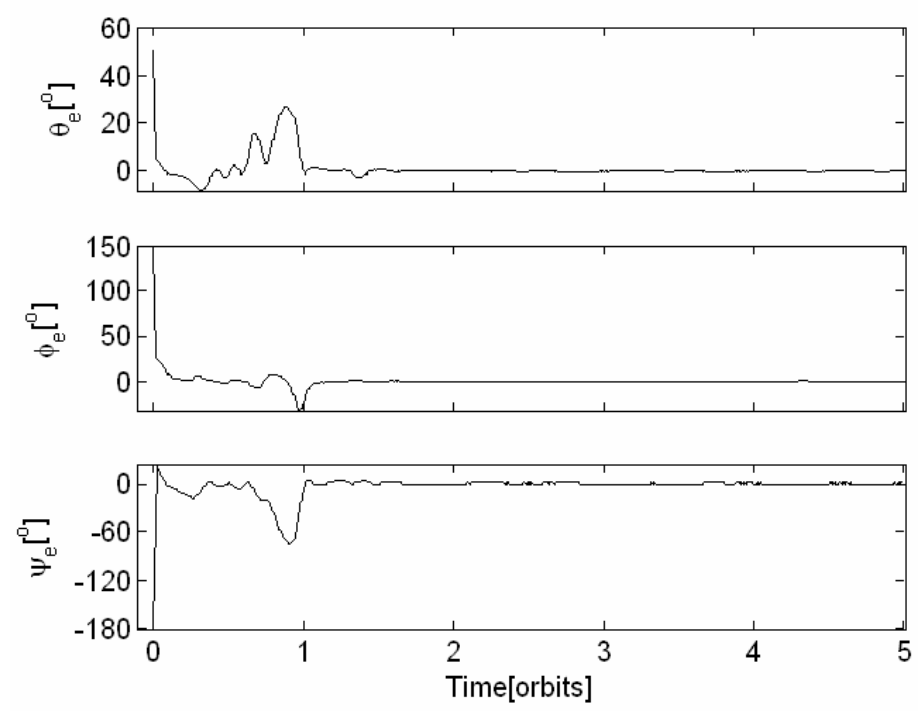

Fig. 1 Average pitch, roll, and yaw angles estimation error.
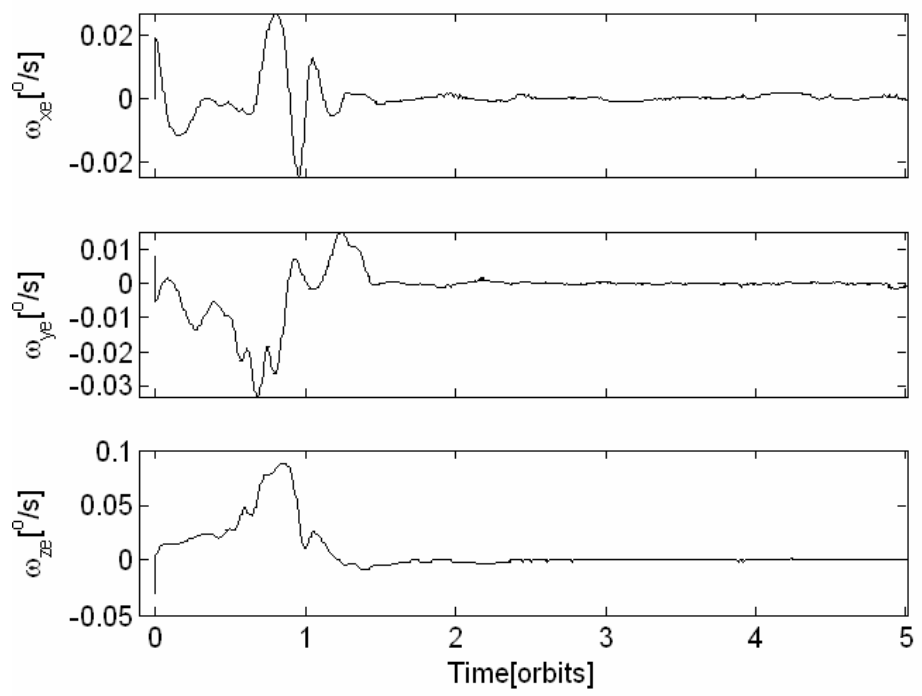

Fig. 2 Average inertial angular velocity Estimation error. 

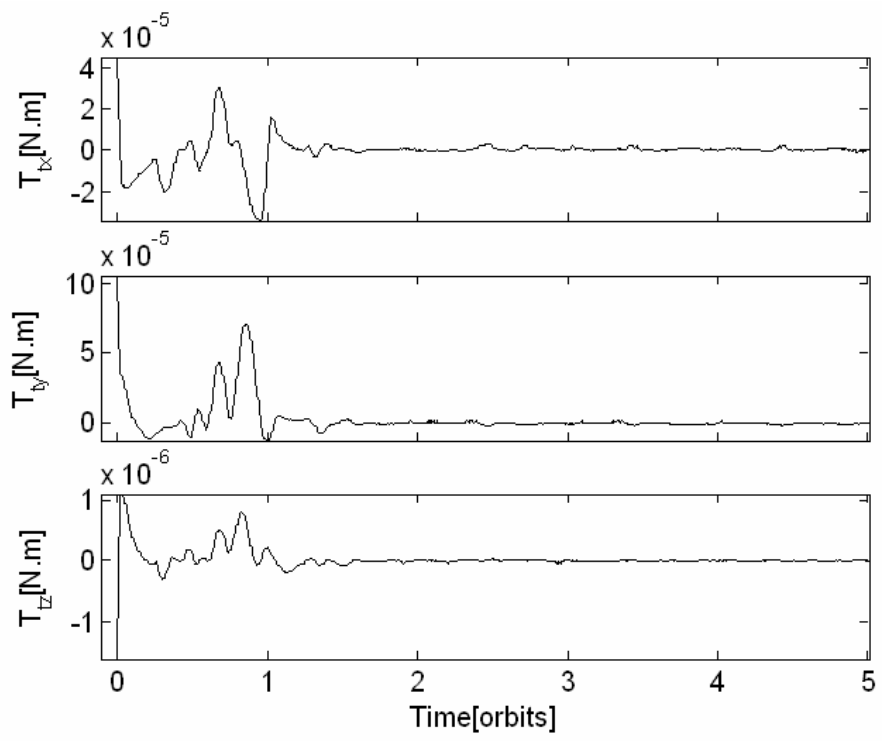

Fig. 3 Average total disturbance moment estimation error.
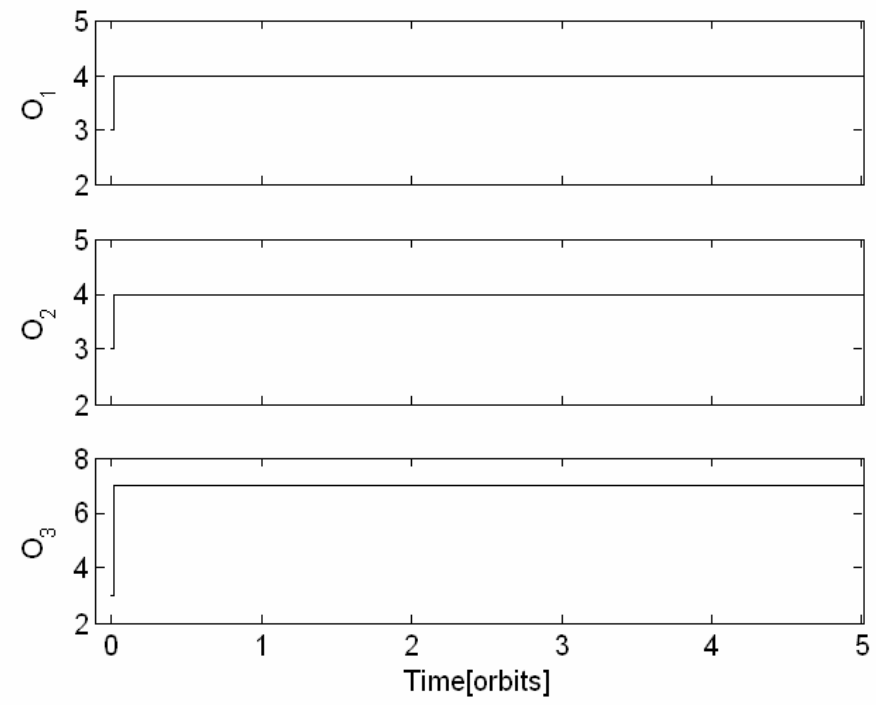

Fig. 4 Rank time history of the observability matrix. 